\title{
Decreased Best Corrected Visual Acuity
}

National Cancer Institute

\section{Source}

National Cancer Institute. Decreased Best Corrected Visual Acuity. NCI Thesaurus. Code C118715.

Diminished clarity of vision despite optimal optical correction (glasses and/or contacts). 